\title{
The holographic supersymmetric Rényi entropy in five dimensions
}

\author{
Luis F. Alday, Paul Richmond and James Sparks \\ Mathematical Institute, University of Oxford, \\ Andrew Wiles Building, Radcliffe Observatory Quarter, \\ Woodstock Road, Oxford, OX2 6GG, U.K. \\ E-mail: alday@maths.ox.ac.uk, richmond@maths.ox.ac.uk, \\ sparks@maths.ox.ac.uk
}

ABSTRACT: We compute the supersymmetric Rényi entropy across an entangling threesphere for five-dimensional superconformal field theories using localization. For a class of $U S p(2 N)$ gauge theories we construct a holographic dual 1/2 BPS black hole solution of Euclidean Romans $F(4)$ supergravity. The large $N$ limit of the gauge theory results agree perfectly with the supergravity computations.

KEYWORDS: Supersymmetry and Duality, Supersymmetric gauge theory, AdS-CFT Correspondence, Supergravity Models

ARXiv EPRINT: 1410.0899 


\section{Contents}

1 Supersymmetric Rényi entropy 1

1.1 Rényi entropy in CFT 1

1.2 Supersymmetry and localization 3

1.3 Large $N$ limit of $U S p(2 N)$ superconformal theories 6

2 Holographic dual $\quad 6$

2.1 Euclidean Romans $F(4)$ supergravity 6

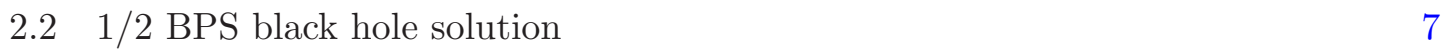

$\begin{array}{lll}2.3 & \text { Global regularity } & 10\end{array}$

$\begin{array}{lll}2.4 & \text { Free energy } & 11\end{array}$

$\begin{array}{lll}2.5 & \text { Wilson loop } & 12\end{array}$

$\begin{array}{lll}3 & \text { Discussion } & 13\end{array}$

\section{Supersymmetric Rényi entropy}

\subsection{Rényi entropy in CFT}

Given a quantum field theory an interesting observable is the Rényi entropy. To define this one divides a spatial slice $\Sigma$ into a region $A$ and its complement $B=\Sigma \backslash A$. The Hilbert space then factorizes

$$
\mathcal{H} \cong \mathcal{H}_{A} \otimes \mathcal{H}_{B}
$$

The reduced density matrix $\rho_{A}$ is defined as

$$
\rho_{A}=\operatorname{Tr}_{B}|0\rangle\langle 0|,
$$

where $|0\rangle$ is the ground state of the theory. For any positive integer $n>1$, the Rényi entropy $S_{n}(A)$ associated to $A$ is then defined as

$$
S_{n}(A)=\frac{1}{1-n} \log \frac{\operatorname{Tr}_{A} \rho_{A}^{n}}{\left(\operatorname{Tr}_{A} \rho_{A}\right)^{n}} .
$$

This is a refinement of the entanglement entropy, which arises by analytically continuing in $n$ and taking the limit

$$
S_{\mathrm{EE}}(A)=\lim _{n \rightarrow 1} S_{n}(A)
$$

One can define the Rényi entropy using the path integral formalism as follows. Consider a Euclidean spacetime with coordinates $\left(t_{E}, x, \vec{z}\right)$, where $t_{E}=\mathrm{i} t$ is the Euclidean time and 
the spatial slice $\Sigma=\left\{t_{E}=0\right\}$. The coordinate $x$ is then defined such that $x \geq 0$ is the region $A$, and $x<0$ its complement $B$. The ground state wave function is given by the path integral

$$
\Psi\left[\psi_{0}(x, \vec{z})\right]=\int_{\left.\psi\right|_{t_{E}=0}=\psi_{0}} \mathcal{D} \psi \mathrm{e}^{-I_{E}(\psi)},
$$

where the fields $\psi$ we are integrating over are defined for negative Euclidean time (or positive imaginary Minkowskian time), and $I_{E}$ is the Euclidean action. The factorization of the $t_{E}=0$ slice into $A \cup B$ leads to a factorization of the boundary data

$$
\psi_{0}(x, \vec{z})= \begin{cases}\psi_{A}(x, \vec{z}) & \text { for } x \geq 0, \\ \psi_{B}(x, \vec{z}) & \text { for } x<0 .\end{cases}
$$

The reduced density matrix is then

$$
\rho_{A}\left(\psi_{A}^{+}, \psi_{A}^{-}\right)=\int \mathcal{D} \psi_{B} \Psi^{\dagger}\left[\psi_{A}^{+}, \psi_{B}\right] \Psi\left[\psi_{A}^{-}, \psi_{B}\right] .
$$

If we let the imaginary time in the two path integral definitions of $\Psi$ run from 0 to $\pm \infty$ respectively, the density matrix becomes the path integral over fields defined on the full Euclidean space, with the $\left(t_{E}, x\right)$ plane cut along the $x>0$ ray and with $\psi_{A}$ taking values $\psi_{A}^{ \pm}$above and below the cut, respectively. The trace of the density matrix is obtained by equating the fields across the cut and carrying out the unrestricted Euclidean path integral. More generally this construction shows that

$$
\operatorname{Tr}_{A} \rho_{A}^{n}=Z_{n},
$$

where $Z_{n}$ is given by the Euclidean path integral over an $n$-sheeted covering of the cut spacetime. This formulation of the Rényi entropy is known as the replica trick [1], and leads to the formula

$$
S_{n}(A)=\frac{1}{1-n} \log \frac{Z_{n}}{\left(Z_{1}\right)^{n}} .
$$

The calculability of $S_{n}(A)$ depends on the choice of spacetime and region $A$. A natural choice is a spacetime of the form $\mathbb{R}_{t_{E}} \times \mathbb{R}^{d-1}=\mathbb{R}^{d}$ and $A$ the unit ball inside $\mathbb{R}^{d-1}$, so that $\partial A=S^{d-2}$. The metric is

$$
\mathrm{d} s_{\mathbb{R}^{d}}^{2}=\mathrm{d} t_{E}^{2}+\mathrm{d} \rho^{2}+\rho^{2} \mathrm{~d} s_{S^{d-2}}^{2},
$$

where $\mathrm{d} s_{S^{d-2}}^{2}$ denotes the round metric on the unit $(d-2)$-sphere. The region $A$ is the ball $0 \leq \rho \leq 1$. For conformal theories it is convenient [2] to perform the computation in the conformally equivalent space $S^{d}$ with metric

$$
\mathrm{d} s_{S^{d}}^{2}=\cos ^{2} \alpha \mathrm{d} \tau^{2}+\mathrm{d} \alpha^{2}+\sin ^{2} \alpha \mathrm{d} s_{S^{d-2}}^{2},
$$

where the change of coordinates is

$$
\begin{aligned}
t_{E} & =\frac{\cos \alpha \sin \tau}{1+\cos \alpha \cos \tau}, \\
\rho & =\frac{\sin \alpha}{1+\cos \alpha \cos \tau} .
\end{aligned}
$$


Here $0 \leq \alpha \leq \pi / 2$ and $\tau$ is periodic with period $2 \pi$. In these coordinates the branch locus is at $\alpha=\pi / 2$ and the cut is at $\tau=0$. In order to compute the Rényi entropy we need to evaluate the partition function on the $n$-branched $d$-sphere, in which case the periodicity of $\tau$ is $2 \pi n$.

The above replica trick, in which one studies field theory on a singular space, is a convenient method to compute entanglement entropies in conformal field theories. However, if one is interested in constructing holographic duals this singularity persists into the bulk, where gravity becomes dynamical. This raises the issue of how to treat the singularity in gravity [3]. An ingenious way to circumvent this problem is to instead conformally map the space to $S^{1} \times \mathbb{H}^{d-1}[4]$

$$
\mathrm{d} s_{S^{1} \times \mathbb{H}^{d-1}}^{2}=\mathrm{d} \tau^{2}+\frac{\mathrm{d} q^{2}}{1+q^{2}}+q^{2} \mathrm{~d} s_{S^{d-2}}^{2},
$$

where $q=\tan \alpha$ takes the range $q \in[0, \infty)$. The coordinates in (1.14) realize the hyperbolic space $\mathbb{H}^{d-1}$ in a spherical slicing. The branch cut at $\alpha=\pi / 2$ has now moved to $q=\infty$. In [4] it was argued that the entanglement entropy maps to a thermal entropy in this space, where the new Euclidean time $\tau$ has period $\beta=2 \pi n$, the inverse temperature. The holographic duals are then naturally black hole solutions with hyperbolic horizons (so called topological black holes).

\subsection{Supersymmetry and localization}

In [5] the authors studied a supersymmetric version of the above Rényi entropy for $\mathcal{N}=2$ supersymmetric theories on the round three-sphere with $d=3$. This is similarly obtained by computing the partition function on $S^{3}$ branched $n$ times over the $S^{1}$ at $\alpha=\pi / 2$, but in addition one needs to turn on an appropriate background R-symmetry gauge field to preserve supersymmetry. After a lengthy computation using localization they find that the partition function $Z_{n}$ is simply the partition function of the squashed sphere $S_{b}^{3}$, with squashing parameter $b=\sqrt{\frac{b_{1}}{b_{2}}}=\sqrt{n}$. In this section we give a simple explanation for this result, which works in general dimensions. Since we will be interested mainly in dimension $d=5$, we shall present the argument for this case.

We write the metric on $S^{5}$ in the form (1.11), where we choose coordinates on $S^{d-2}=$ $S^{3}$ as

$$
\mathrm{d} s_{S^{3}}^{2}=\mathrm{d} \theta^{2}+\cos ^{2} \theta \mathrm{d} \psi^{2}+\sin ^{2} \theta \mathrm{d} \phi^{2} .
$$

Here $\psi$ and $\phi$ both have period $2 \pi$, while $0 \leq \theta \leq \pi / 2$. In order to define supersymmetric field theories on $S^{5}$ (or its branching along $S^{3}$ ) one needs to choose a Killing spinor $\epsilon$. The Killing spinors on $S^{5}$ have charges $\pm 1 / 2$ under the Lie derivatives along $\partial_{\tau}, \partial_{\psi}, \partial_{\phi}$, which generate a $\mathrm{U}(1)^{3} \subset \mathrm{SO}(6)$ subset of isometries. In particular our choice of spinor will be such that

$$
\mathcal{L}_{\partial_{\tau}} \epsilon=-\frac{\mathrm{i}}{2} \epsilon .
$$

This charge guarantees that the spinor $\epsilon$ is smooth at $\alpha=\pi / 2$, where $\partial_{\tau}=0$. Indeed, the normal space to $\alpha=\pi / 2$ is a copy of $\mathbb{R}^{2} \cong \mathbb{C}$. One can then introduce a polar radial 
variable $R=\pi / 2-\alpha$, and corresponding Cartesian coordinates $X=R \cos \tau, Y=R \sin \tau$ on this normal space. The frame $e_{1}=\mathrm{d} X, e_{2}=\mathrm{d} Y$ rotates with charge 1 under $\partial_{\tau}$, so that positive and negative chirality spinors in $\mathbb{R}^{2}$ correspondingly rotate with charge $\pm 1 / 2$, respectively. One could instead move to the non-rotating frame $\hat{e}_{1}=\mathrm{d} R, \hat{e}_{2}=R \mathrm{~d} \tau$, in which the spinor will then have an explicit overall phase $\mathrm{e}^{-\mathrm{i} \tau / 2}$. However, this frame is singular at the origin $R=0$ which is why the spinor looks singular there.

Similarly, we choose conventions so that $\epsilon$ has charge $-1 / 2$ under $\partial_{\psi}$ and $+1 / 2$ under $\partial_{\phi}$. The vector bilinear $K^{\mu}=\epsilon^{\dagger} \gamma^{\mu} \epsilon$ is then the Killing vector

$$
K=-\partial_{\phi}+\partial_{\psi}+\partial_{\tau},
$$

which generates a Hopf foliation of $S^{5}$.

To form the $n$-branched sphere one simply takes $\tau$ to have period $2 \pi n$. We may introduce a local complex coordinate $w=R \mathrm{e}^{\mathrm{i} \tau}$ on the normal space $\mathbb{R}^{2}$ to the branch locus $\alpha=\pi / 2$. Then $z=w^{1 / n}=R^{1 / n} \mathrm{e}^{\mathrm{i} \varphi_{3}}$ has $\arg z=\varphi_{3}=\tau / n$, which has the canonical period $2 \pi$. Moreover, a function is smooth at the branch point $R=0$ means that it is smooth in the coordinate $z$. For example, when we come to discuss the computation of one-loop determinants below, it is convenient to expand in Fourier modes of the $\left(S^{1}\right)^{3}=\mathrm{U}(1)^{3}$ isometry, and a complete set of modes in the $\tau$ direction is then $\mathrm{e}^{\mathrm{i} m_{3} \varphi_{3}}$, with $m_{3} \in \mathbb{Z}$.

The Killing spinor $\epsilon$ on the round sphere has charge $-\mathrm{in} / 2$ under $\partial_{\varphi_{3}}$, and is thus singular along the branch locus when $n>1$. We may remedy this, as in [5], by introducing the background R-symmetry gauge field

$$
\mathcal{A}=-\frac{n-1}{n} \mathrm{~d} \tau=-(n-1) \mathrm{d} \varphi_{3}
$$

In a five-dimensional supersymmetric gauge theory we view this as embedded in $\mathrm{U}(1)_{R} \subset$ $\mathrm{SU}(2)_{R}$, where the gauge covariant derivative on $\epsilon$ is ${ }^{1}$

$$
D_{\mu} \epsilon=\nabla_{\mu} \epsilon+\frac{\mathrm{i}}{2} \mathcal{A}_{\mu} \epsilon
$$

The flat gauge field (1.18) is smooth everywhere on the branched sphere, except at the branch locus $\alpha=\pi / 2$ where $\partial_{\tau}=0$. This singularity is designed precisely so as to render the resulting Killing spinor smooth. To see this, note that we may write $\mathcal{A}=\mathrm{i} g^{-1}(\mathrm{~d} g)=$ id $\log g$, where

$$
g=\mathrm{e}^{\mathrm{i}(n-1) \tau / n} .
$$

The factor of $1 / 2$ in (1.19) is chosen to match our Romans supergravity conventions in the next section, but in particular this implies that the spinor transforms as

$$
\epsilon_{\text {branched }}=g^{1 / 2} \epsilon \text {. }
$$

Of course then

$$
D_{\mu} \epsilon_{\text {branched }}=g^{1 / 2} \nabla_{\mu} \epsilon,
$$

\footnotetext{
${ }^{1}$ The second spinor in the $\mathrm{SU}(2)_{R}$ doublet then has the opposite charge under $\mathcal{A}_{\mu}$.
} 
so that $\epsilon_{\text {branched }}$ satisfies the same Killing spinor as on the round sphere, but with the Levi-Civita spin connection replaced by gauge-covariant derivative (1.19). Moreover, in a non-rotating frame for $\partial_{\tau}$, the $\tau$-dependent phase of the new spinor is

$$
\mathrm{e}^{-\mathrm{i} \tau / 2} \cdot \mathrm{e}^{\mathrm{i}(n-1) \tau / 2 n}=\mathrm{e}^{-\mathrm{i} \tau / 2 n}=\mathrm{e}^{-\mathrm{i} \phi_{3} / 2} .
$$

This shows that the charged spinor $\epsilon_{\text {branched }}$ is non-singular at the branch locus, and hence non-singular everywhere on the branched five-sphere. Moreover, since the Killing vector bilinear $K^{\mu}=\epsilon_{\text {branched }}^{\dagger} \gamma^{\mu} \epsilon_{\text {branched }}$ is the same as that for the uncharged spinor $\epsilon$, we have from (1.17)

$$
K=-\partial_{\phi}+\partial_{\psi}+\partial_{\tau}=b_{1} \partial_{\varphi_{1}}+b_{2} \partial_{\varphi_{2}}+b_{3} \partial_{\varphi_{3}}
$$

Here we have introduced the angular coordinates

$$
\varphi_{1}=-\phi, \quad \varphi_{2}=\psi, \quad \varphi_{3}=\frac{1}{n} \tau,
$$

on $\mathrm{U}(1)^{3}$, which all have canonical $2 \pi$ periods, and $\left(b_{1}, b_{2}, b_{3}\right)=\left(1,1, \frac{1}{n}\right)$.

Imagine now computing the perturbative partition function of a supersymmetric field theory on the $n$-branched five-sphere using localization. Locally this computation is the same as that on the round sphere $[8,9]$. What changes are the boundary conditions along the branch locus at $\alpha=\pi / 2$. However, as explained above, these boundary conditions simply mean that fields are smooth in the $z$ coordinate, rather than the original $w$ coordinate. In particular, one expands in Fourier modes exp $\left[\mathrm{i}\left(m_{1} \varphi_{1}+m_{2} \varphi_{2}+m_{3} \varphi_{3}\right)\right]$ where $m_{i} \in \mathbb{Z}$, $i=1,2,3$. The charge of such a mode under the supersymmetric Killing vector $K$ is then $m_{1} b_{1}+m_{2} b_{2}+m_{3} b_{3}$. The Killing spinor $\epsilon_{\text {branched }}$ itself similarly has charge $-\left(b_{1}+b_{2}+b_{3}\right) / 2$. Combining these observations with the structure of the one-loop calculations in three dimensions in $[10,11]$ then leads immediately to the result in [5]: the partition function $Z_{n}$ is simply the partition function of any three-sphere background with $\left(b_{1}, b_{2}\right)=\left(1, \frac{1}{n}\right)$. In particular most modes in the one-loop determinant pair under supersymmetry, so that their contributions cancel. For the remaining unpaired modes, their eigenvalues depend on the background geometry only via their charge $m_{1} b_{1}+m_{2} b_{2}$. The determinant over normalizable modes then leads to a double sine function $S_{2}\left(\cdot \mid\left(b_{1}, b_{2}\right)\right)$. In five dimensions, similar reasoning applies to the explicit computation of the perturbative partition function on $S^{5}$. We then expect the result

$$
Z_{n}^{\text {pert }}=C(\mathbf{b}) \prod_{a=1}^{\text {rank } G} \int_{-\infty}^{\infty} \mathrm{d} \sigma_{a} \mathrm{e}^{-\frac{(2 \pi)^{3}}{b_{1} b_{2} b_{3}} \mathscr{F}(\sigma)} \frac{\prod_{\alpha} S_{3}(-\mathrm{i} \alpha(\sigma) \mid \mathbf{b})}{\prod_{\rho} S_{3}\left(-\mathrm{i} \rho(\sigma)+\frac{1}{2}\left(b_{1}+b_{2}+b_{3}\right) \mid \mathbf{b}\right)} .
$$

Here the prefactor $C(\mathbf{b})$ depends only on $\mathbf{b}=\left(b_{1}, b_{2}, b_{3}\right)=\left(1,1, \frac{1}{n}\right)$, and in particular will not contribute to the large $N$ limit of interest in the next subsection. The product over $\alpha$ in the numerator is over roots of the gauge group $G$, while the product over $\rho$ in the denominator is over weights in a weight space decomposition of the matter representation R. The integral in $\sigma_{a}$ is over the Cartan of $G, \mathscr{F}$ is the prepotential of the theory, while $S_{3}(\cdot \mid \mathbf{b})$ is the triple sine function. The result (1.26) also agrees with the conjecture the 
authors made in $[12,13]$ : that the partition function for any supersymmetric five-sphere background depends on the background only via the Killing vector $K$. In particular, (1.26) equals the squashed five-sphere perturbative partition function [14].

\subsection{Large $N$ limit of $U S p(2 N)$ superconformal theories}

The result for the perturbative partition function (1.26) is valid for a general supersymmetric gauge theory in five dimensions. We now focus on a particular class of theories with gauge group $G=U S p(2 N)$ and matter consisting of $N_{f}$ hypermultiplets in the fundamental and a single hypermultiplet in the anti-symmetric representation of $G$. These theories arise from a system of $N$ D4-branes and some number of D8-branes and orientifold planes in massive type IIA string theory, and have a large $N$ limit that has a dual description in massive type IIA supergravity [15-17]. For these theories, the large $N$ limit of (1.26) gives the free energy [13]

$$
\begin{aligned}
F=-\log Z_{n}^{\text {pert }} & =\frac{\left(b_{1}+b_{2}+b_{3}\right)^{3}}{27 b_{1} b_{2} b_{3}} F_{S_{\text {round }}^{5}} \\
& =\frac{(1+2 n)^{3}}{27 n^{2}} F_{S_{\text {round }}^{5}} .
\end{aligned}
$$

Here $F_{S_{\text {round }}^{5}}=\frac{9 \sqrt{2} \pi N^{5 / 2}}{5 \sqrt{8-N_{f}}}+\mathcal{O}\left(N^{3 / 2}\right)$ is the large $N$ limit of the free energy on the round five-sphere computed in reference [18]. This results in the following large $N$ Rényi entropy

$$
S_{n}=S_{n}\left(S^{3}\right)=-\frac{1+7 n+19 n^{2}}{27 n^{2}} F_{S_{\text {round }}^{5}} .
$$

In the next section we will reproduce this result from the holographic dual computation.

\section{Holographic dual}

Following [4], and similar computations in lower dimensions [19-22], the holographic supersymmetric Rényi entropy is computed from a 1/2 BPS Euclidean black hole solution. As explained in [13], we may construct this dual solution in Euclidean Romans F(4) supergravity, and then uplift this to a solution of massive IIA string theory.

\subsection{Euclidean Romans $F(4)$ supergravity}

The bosonic fields of the six-dimensional Romans supergravity theory [23] consist of the metric, a scalar field $X$, a two-form potential $B$, and a one-form potential $A$, together with an $\mathrm{SO}(3) \sim \mathrm{SU}(2)$ gauge field $A^{i}$ where $i=1,2,3$. For the solution of interest in this paper the two-form potential vanishes, $B=0$, and we work in a gauge in which the Stueckelberg one-form $A$ is zero. Setting also the gauge coupling constant to unity, the Euclidean equations of motion are $[12,13]$

$$
\begin{aligned}
F^{i} \wedge F^{i} & =0 \\
D\left(X^{-2} * F^{i}\right) & =0 \\
\mathrm{~d}\left(X^{-1} * \mathrm{~d} X\right) & =-\left(\frac{1}{6} X^{-6}-\frac{2}{3} X^{-2}+\frac{1}{2} X^{2}\right) * 1-\frac{1}{8} X^{-2}\left(F^{i} \wedge * F^{i}\right) .
\end{aligned}
$$


The first equation is a remnant of the $B$-field equation of motion, and $D \omega^{i}=\mathrm{d} \omega^{i}-\epsilon_{i j k} A^{j} \wedge$ $\omega^{k}$ is the $\mathrm{SO}(3)$ covariant derivative. The Einstein equation is

$$
\begin{aligned}
R_{\mu \nu}= & 4 X^{-2} \partial_{\mu} X \partial_{\nu} X+\left(\frac{1}{18} X^{-6}-\frac{2}{3} X^{-2}-\frac{1}{2} X^{2}\right) g_{\mu \nu} \\
& +\frac{1}{2} X^{-2}\left(\left(F^{i}\right)_{\mu \nu}^{2}-\frac{1}{8}\left(F^{i}\right)^{2} g_{\mu \nu}\right)
\end{aligned}
$$

where $\left(F^{i}\right)_{\mu \nu}^{2}=F_{\mu \rho}^{i} F_{\nu}^{i \rho}$. The Euclidean action is

$$
\begin{gathered}
I_{E}=-\frac{1}{16 \pi G_{6}} \int_{M_{6}} R * 1-4 X^{-2}\left(\mathrm{~d} X \wedge * \mathrm{~d} X+\frac{1}{8} F^{i} \wedge * F^{i}\right) \\
-\left(\frac{2}{9} X^{-6}-\frac{8}{3} X^{-2}-2 X^{2}\right) * 1 .
\end{gathered}
$$

A solution to the above equations of motion is supersymmetric provided there exist nontrivial Dirac spinors $\epsilon_{I}, I=1,2$, satisfying the following Killing spinor and dilatino equation

$$
\begin{aligned}
D_{\mu} \epsilon_{I} & =\frac{\mathrm{i}}{4 \sqrt{2}}\left(X+\frac{1}{3} X^{-3}\right) \Gamma_{\mu} \Gamma_{7} \epsilon_{I}+\frac{1}{16 \sqrt{2}} X^{-1} F_{\nu \rho}^{i}\left(\Gamma_{\mu}^{\nu \rho}-6 \delta_{\mu}^{\nu} \Gamma^{\rho}\right) \Gamma_{7}\left(\sigma^{i}\right)_{I}^{J} \epsilon_{J}, \\
0 & =-\mathrm{i} X^{-1} \partial_{\mu} X \Gamma^{\mu} \epsilon_{I}+\frac{1}{2 \sqrt{2}}\left(X-X^{-3}\right) \Gamma_{7} \epsilon_{I}-\frac{\mathrm{i}}{8 \sqrt{2}} X^{-1} F_{\mu \nu}^{i} \Gamma^{\mu \nu} \Gamma_{7}\left(\sigma^{i}\right)_{I}{ }^{J} \epsilon_{J} .
\end{aligned}
$$

Here $\Gamma_{\mu}$ generate the Clifford algebra $\operatorname{Cliff}(6,0)$ in an orthonormal frame, and we have defined the chirality operator $\Gamma_{7}=\mathrm{i} \Gamma_{012345}$, which satisfies $\left(\Gamma_{7}\right)^{2}=1$. The covariant derivative acting on the spinor is $D_{\mu} \epsilon_{I}=\nabla_{\mu} \epsilon_{I}+\frac{i}{2} A_{\mu}^{i}\left(\sigma^{i}\right)_{I}^{J} \epsilon_{J}$.

\section{$2.21 / 2$ BPS black hole solution}

Our starting point is the charged AdS black hole solution of [24]. After a Wick rotation and a relabelling of parameters, the solution is

$$
\mathrm{d} s^{2}=\frac{H(r)^{1 / 2}}{f(r)} \mathrm{d} r^{2}+\frac{9 f(r)}{2 H(r)^{3 / 2}} \mathrm{~d} \tau^{2}+r^{2} H(r)^{1 / 2} \mathrm{~d} s_{\mathbb{H}^{4}}^{2},
$$

where

$$
\begin{aligned}
H(r) & =1+\frac{Q}{r^{3}} \\
f(r) & =-1-\frac{\gamma}{r^{3}}+\frac{2}{9} r^{2} H(r)^{2} .
\end{aligned}
$$

The solution depends on the two parameters $Q$ and $\gamma$, and $\mathrm{d} s_{\mathbb{H}^{4}}^{2}$ is the metric of a unit radius hyperbolic space. As in section 1 we choose coordinates so that

$$
\mathrm{d} s_{\mathbb{H}^{4}}^{2}=\frac{1}{\left(1+q^{2}\right)} \mathrm{d} q^{2}+q^{2}\left(\mathrm{~d} \theta^{2}+\cos ^{2} \theta \mathrm{d} \psi^{2}+\sin ^{2} \theta \mathrm{d} \phi^{2}\right) .
$$

$c f$. equations (1.14), (1.15). The remaining fields are

$$
\begin{aligned}
X(r) & =H(r)^{-1 / 4} \\
\mathcal{A} & \equiv A^{3}=3 \sqrt{1-\frac{\gamma}{Q} \frac{H(r)-1}{H(r)} \mathrm{d} \tau+\mu \mathrm{d} \tau .}
\end{aligned}
$$


Notice that the parameter $Q$ is necessarily non-zero if $\gamma \neq 0$. We have also added a pure gauge term $\mu \mathrm{d} \tau$ to $\mathcal{A}$, which as we shall see is required in order that the gauge field is non-singular at the horizon.

The metric (2.6) is asymptotically locally AdS for large $r$. Specifically

$$
\mathrm{d} s^{2} \simeq \frac{9 \mathrm{~d} r^{2}}{2 r^{2}}+r^{2}\left(\mathrm{~d} \tau^{2}+\mathrm{d} s_{\mathbb{H}^{4}}^{2}\right)
$$

to leading order as $r \rightarrow \infty$. Moreover, the scalar field $X \rightarrow 1$ while $\mathcal{A} \rightarrow \mu \mathrm{d} \tau$. Since $\tau$ will be periodically identified in the next subsection, the conformal boundary geometry is $S^{1} \times \mathbb{H}^{4}$.

The solution is supported by a single component of the $\mathrm{SU}(2)$ gauge field, and without loss of generality we have chosen this to lie along the $i=3$ direction. For this choice of gauge the Killing spinor equations for $\epsilon_{1}$ and $\epsilon_{2}$ decouple. Moreover, if the fields are all real then the Killing spinor equation for $\epsilon_{2}$ is simply the charge conjugate of that for $\epsilon_{1}$ [13]. Hence we can consider only the spinor $\epsilon=\epsilon_{1}$ which satisfies

$$
\begin{aligned}
D_{\mu} \epsilon & =\frac{\mathrm{i}}{4 \sqrt{2}}\left(X+\frac{1}{3} X^{-3}\right) \Gamma_{\mu} \Gamma_{7} \epsilon+\frac{1}{16 \sqrt{2}} X^{-1} \mathcal{F}_{\nu \rho}\left(\Gamma_{\mu}{ }^{\nu \rho}-6 \delta_{\mu}{ }^{\nu} \Gamma^{\rho}\right) \Gamma_{7} \epsilon, \\
0 & =-\mathrm{i} X^{-1} \partial_{\mu} X \Gamma^{\mu} \epsilon+\frac{1}{2 \sqrt{2}}\left(X-X^{-3}\right) \Gamma_{7} \epsilon-\frac{\mathrm{i}}{8 \sqrt{2}} X^{-1} \mathcal{F}_{\mu \nu} \Gamma^{\mu \nu} \Gamma_{7} \epsilon,
\end{aligned}
$$

with $\mathcal{F}=\mathrm{d} \mathcal{A}$.

The above black hole solution is $1 / 2$ BPS for $\gamma=0$. To see this we introduce the frame

$$
\begin{aligned}
& e^{0}=\frac{H(r)^{1 / 4}}{f(r)^{1 / 2}} \mathrm{~d} r, \quad e^{1}=\frac{3}{\sqrt{2}} \frac{f(r)^{1 / 2}}{H(r)^{3 / 4}} \mathrm{~d} \tau, \quad e^{2}=\frac{r H(r)^{1 / 4}}{\left(1+q^{2}\right)^{1 / 2}} \mathrm{~d} q, \\
& e^{3}=q r H(r)^{1 / 4} \mathrm{~d} \theta, \quad e^{4}=q r H(r)^{1 / 4} \cos \theta \mathrm{d} \psi, \quad e^{5}=q r H(r)^{1 / 4} \sin \theta \mathrm{d} \phi,
\end{aligned}
$$

and the following basis of six-dimensional gamma matrices

$$
\begin{aligned}
\Gamma_{0} & =\left(\begin{array}{cc}
0 & 1_{4} \\
1_{4} & 0
\end{array}\right), \quad \Gamma_{m}=\left(\begin{array}{cc}
0 & \mathrm{i} \gamma_{m} \\
-\mathrm{i} \gamma_{m} & 0
\end{array}\right), \quad m=1, \ldots, 5, \\
\Gamma_{7} & =\left(\begin{array}{cc}
-1_{4} & 0 \\
0 & 1_{4}
\end{array}\right)
\end{aligned}
$$

where $1_{4}$ is the $4 \times 4$ unit matrix and $\gamma_{m}$ are a basis for Cliff $(5,0)$. In this basis the dilatino condition (2.12) can be written as

$$
M \epsilon=0,
$$

where $M$ is an $8 \times 8$ matrix. A necessary condition to have a non-trivial Killing spinor is $\operatorname{det} M=0$. We compute

$$
\operatorname{det} M=\frac{3^{8}}{2^{16}} \frac{Q^{4} \gamma^{4}}{r^{14}\left(r^{3}+Q\right)^{6}},
$$


from which we conclude that $\gamma=0$ is necessary for supersymmetry. In order to show that $\gamma=0$ is also sufficient, we next directly solve the Killing spinor equation (2.11). Defining

$$
\begin{aligned}
& f_{1}(r)=\frac{r^{1 / 8} \sqrt{3 \sqrt{2} r^{2}+2 r^{3}+2 Q}}{\left(r^{3}+Q\right)^{3 / 8}}, \\
& f_{2}(r)=\frac{r^{1 / 8} \sqrt{-3 \sqrt{2} r^{2}+2 r^{3}+2 Q}}{\left(r^{3}+Q\right)^{3 / 8}},
\end{aligned}
$$

the general solution to the dilatino and Killing spinor equation takes the form

$$
\epsilon=\sqrt{1+\sqrt{1+q^{2}}}\left(\begin{array}{c}
\mathrm{e}^{-\frac{1}{2} \mathrm{i}(\tau+\theta+\phi+\psi)}\left(\mathrm{e}^{\mathrm{i} \phi} \kappa_{1}+\mathrm{e}^{\mathrm{i} \psi} \kappa_{2}\right) f_{1}(r) \\
\mathrm{ie}^{-\frac{1}{2} \mathrm{i}(\tau-\theta+\phi+\psi)}\left(\mathrm{e}^{\mathrm{i} \phi} \kappa_{1}-\mathrm{e}^{\mathrm{i} \psi} \kappa_{2}\right) f_{1}(r) \\
\mathrm{e}^{-\frac{1}{2} \mathrm{i}(\tau-\theta+\phi+\psi)}\left(\mathrm{e}^{\mathrm{i}(\phi+\psi)} \kappa_{3}+\kappa_{4}\right) f_{2}(r) \\
\mathrm{ie}^{-\frac{1}{2} \mathrm{i}(\tau+\theta+\phi+\psi)}\left(\mathrm{e}^{\mathrm{i}(\phi+\psi)} \kappa_{3}-\kappa_{4}\right) f_{2}(r) \\
-\mathrm{ie}^{-\frac{1}{2} \mathrm{i}(\tau+\theta+\phi+\psi)}\left(\mathrm{e}^{\mathrm{i} \phi} \kappa_{1}+\mathrm{e}^{\mathrm{i} \psi} \kappa_{2}\right) f_{2}(r) \\
\mathrm{e}^{-\frac{1}{2} \mathrm{i}(\tau-\theta+\phi+\psi)}\left(\mathrm{e}^{\mathrm{i} \phi} \kappa_{1}-\mathrm{e}^{\mathrm{i} \psi} \kappa_{2}\right) f_{2}(r) \\
-\mathrm{e}^{-\frac{1}{2} \mathrm{i}(\tau-\theta+\phi+\psi)}\left(\mathrm{e}^{\mathrm{i}(\phi+\psi)} \kappa_{3}+\kappa_{4}\right) f_{1}(r) \\
\mathrm{e}^{-\frac{1}{2} \mathrm{i}(\tau+\theta+\phi+\psi)}\left(\mathrm{e}^{\mathrm{i}(\phi+\psi)} \kappa_{3}-\kappa_{4}\right) f_{1}(r)
\end{array}\right)
$$

The four integration constants $\kappa_{a}, a=1,2,3,4$, show that the solution preserves half of the maximal 8 supercharges.

When the supergravity fields are all real the vector field

$$
K^{\mu}=\epsilon^{\dagger} \Gamma^{\mu} \epsilon
$$

is Killing [13]. In the case at hand we obtain a family of Killing vectors, depending on the integration constants $\kappa_{a}$. For generic values of the parameter $Q$ the black hole solution has symmetry $\mathrm{U}(1)_{\tau} \times \mathrm{SO}(4,1)$, where $\mathrm{SO}(4,1)$ is the isometry group of $\mathbb{H}^{4}$. In particular this contains the maximal torus $\mathrm{U}(1)^{3} \subset \mathrm{U}(1)_{\tau} \times \mathrm{SO}(4,1)$. By choosing the integration constants $\kappa_{a}$ as

$$
\kappa_{1}=\frac{1}{2 \sqrt{2}}, \quad \kappa_{2}=\kappa_{3}=\kappa_{4}=0
$$

the Killing vector (2.19) can be chosen to lie in the Lie algebra of this maximal torus. Explicitly, we find

$$
K=-\partial_{\phi}+\partial_{\psi}+\partial_{\tau}
$$




\subsection{Global regularity}

In order to have a globally regular supergravity solution we must in particular check that the Euclidean black hole (2.6) smoothly closes off at the horizon. This occurs at the largest root $r_{h}>0$ of the function $f(r)$. Imposing $f\left(r_{h}\right)=0$ leads to the relation

$$
Q=r_{h}^{2}\left(\frac{3}{\sqrt{2}}-r_{h}\right)
$$

When $Q=0$ we note that the metric (2.6) is simply Euclidean $\mathrm{AdS}_{6}$, written in a hyperbolic slicing, and $r_{h}=\frac{3}{\sqrt{2}}=\ell$ is the AdS radius.

In general, near to $r=r_{h}$ the metric is to leading order

$$
\mathrm{d} s^{2} \simeq \mathrm{d} R^{2}+\left(\sqrt{2} r_{h}-2\right)^{2} R^{2} \mathrm{~d} \tau^{2}+H\left(r_{h}\right)^{1 / 2} r_{h}^{2} \mathrm{~d} s_{\mathbb{H}^{4}}^{2},
$$

where we have defined the new radial coordinate

$$
R=2^{3 / 8} 3^{1 / 4} \frac{r_{h}^{1 / 4}}{\left(\sqrt{2} r_{h}-2\right)^{1 / 2}}\left(r-r_{h}\right)^{1 / 2} .
$$

We see that the space smoothly closes off at the horizon $R=0$ provided $\tau$ has period $\beta$, where

$$
\beta=\frac{2 \pi}{\sqrt{2} r_{h}-2} .
$$

Comparing to section 1 , where $\tau$ has period $2 \pi n$ with $n$ the replica index, we see that $\beta=2 \pi n$ and

$$
r_{h}=\frac{1+2 n}{\sqrt{2} n} .
$$

Notice that $n=1$ gives the Euclidean $\mathrm{AdS}_{6}$ solution with $Q=0$.

Similarly, in order that the gauge field $\mathcal{A}$ in (2.9) is non-singular at the horizon we have

$$
3 \frac{H\left(r_{h}\right)-1}{H\left(r_{h}\right)}+\mu=0
$$

which using (2.26) becomes

$$
\mu=-\frac{(n-1)}{n} .
$$

Thus the restriction of $\mathcal{A}$ to the conformal boundary gives

$$
\left.\mathcal{A}\right|_{r=\infty}=\mu \mathrm{d} \tau=-\frac{(n-1)}{n} \mathrm{~d} \tau .
$$

Note that this agrees with the R-symmetry gauge field (1.18) required for supersymmetry on the $n$-branched sphere. 
The resulting supergravity solution is then smooth, with the global topology being a product of $\mathbb{R}^{2}$ with $\mathbb{H}^{4} \cong \mathbb{R}^{4}$, with the origin of $\mathbb{R}^{2}$ being the horizon at $r=r_{h}$. Thus the solution is defined on $\mathbb{R}^{6}$, with the action of the maximal torus $\mathrm{U}(1)^{3} \subset \mathrm{U}(1)_{\tau} \times$ $\mathrm{SO}(4,1)$ making this naturally into $\mathbb{R}^{6} \cong \mathbb{R}^{2} \oplus \mathbb{R}^{2} \oplus \mathbb{R}^{2}$. Introducing standard $2 \pi$ period coordinates (1.25), the Killing vector bilinear (2.21) becomes

$$
K=b_{1} \partial_{\varphi_{1}}+b_{2} \partial_{\varphi_{2}}+b_{3} \partial_{\varphi_{3}}
$$

where $\left(b_{1}, b_{2}, b_{3}\right)=\left(1,1, \frac{1}{n}\right)$. Also notice that the restriction of this vector to the conformal boundary at $r=\infty$ agrees with the supersymmetric Killing vector in section 1.2.

\section{$2.4 \quad$ Free energy}

The holographic free energy is computed by evaluating the renormalized on-shell action. This takes the form

$$
F=I_{\text {ren }}=I_{E}+I_{\mathrm{GH}}+I_{\text {counterterms }}
$$

Here $I_{E}$ is the Euclidean supergravity action (2.3). The Gibbons-Hawking boundary term is

$$
I_{\mathrm{GH}}=-\frac{1}{8 \pi G_{6}} \int_{\partial M_{6}} \mathcal{K} \sqrt{\operatorname{det} h} \mathrm{~d}^{5} x,
$$

where the space $M_{6}$ has boundary $\partial M_{6}, h_{m n}$ is the induced metric and $\mathcal{K}$ denotes the trace of the second fundamental form. The boundary counterterms for the general sixdimensional Euclidean Romans $F(4)$ theory were first given in $[12,13]$. For the present case the two-form potential $B=0$, and consequently the general counterterm expression simplifies greatly to

$$
\begin{aligned}
I_{\text {counterterms }}=\frac{1}{8 \pi G_{6}} \int_{\partial M_{6}} & {\left[\frac{4 \sqrt{2}}{3}+\frac{1}{2 \sqrt{2}} R(h)+\frac{3}{4 \sqrt{2}} R(h)_{m n} R(h)^{m n}-\frac{15}{64 \sqrt{2}} R(h)^{2}\right.} \\
& \left.-\frac{3}{4 \sqrt{2}}\|\mathcal{F}\|_{h}^{2}+\frac{4 \sqrt{2}}{3}(1-X)^{2}\right] \sqrt{\operatorname{det} h} \mathrm{~d}^{5} x,
\end{aligned}
$$

where $R(h)_{m n}, R(h)$ are respectively the Ricci tensor and scalar of the induced metric. For the Euclidean black hole solution of interest the restriction of the field strength $\mathcal{F}$ to the conformal boundary is zero.

In order to compute the regularized free energy we cut off the radial coordinate at $r=\Lambda$ :

$$
F=\lim _{\Lambda \rightarrow \infty}\left[I_{E}(\Lambda)+I_{\mathrm{GH}}(\Lambda)+I_{\text {counterterms }}(\Lambda)\right],
$$

where the relevant integrals are over $M_{6}(\Lambda)$ and $\partial M_{6}(\Lambda)$, respectively. For our black hole solution the integrals over $\tau$ and the hyperbolic space $\mathbb{H}^{4}$ factorize, so that the former contributes $2 \pi n$ to the integral, while the latter contributes a factor of $\operatorname{vol}\left(\mathbb{H}^{4}\right)$. The integral over the radial variable $r$ is then easily evaluated in (2.34), and we obtain

$$
F=-\frac{3 n}{4 \sqrt{2} G_{6}} \operatorname{vol}\left(\mathbb{H}^{4}\right) r_{h}^{3}
$$


The volume $\operatorname{vol}\left(\mathbb{H}^{4}\right)$ is divergent. However, one can also regularize this using boundary counterterms (notice that $\mathbb{H}^{4}$ is Euclidean $\mathrm{AdS}_{4}$ ). Doing so one obtains

$$
\operatorname{vol}\left(\mathbb{H}^{4}\right)=\frac{4 \pi^{2}}{3}
$$

Substituting for the horizon radius $r_{h}$ in terms of $n$ (2.26), the final formula for the free energy is

$$
F=F_{n}=\frac{(1+2 n)^{3}}{27 n^{2}} F_{1}
$$

where $F_{1}$ agrees with the free energy of Euclidean $\mathrm{AdS}_{6}$ in a round $S^{5}$ slicing. This agrees precisely with (1.27).

\subsection{Wilson loop}

As explained in [24], solutions of the Euclidean Romans supergravity theory uplift to solutions of massive type IIA supergravity, of the warped product form $M_{6} \times S^{4}$. In [25] the holographic dual of a BPS Wilson loop in the fundamental representation was argued to be a fundamental string, sitting at the pole of $S^{4}$. Here the boundary superconformal field theories are the $U S p(2 N)$ gauge theories discussed in section 1.3. In [13] the string action for a general background was shown to be

$$
S_{\text {string }}=\frac{5 \pi}{4 N^{2} G_{6}}\left[\int_{\Sigma_{2}}\left(X^{-2} \sqrt{\operatorname{det} \gamma} \mathrm{d}^{2} x+\mathrm{i} B\right)-\frac{3}{\sqrt{2}} \operatorname{length}\left(\partial \Sigma_{2}\right)\right] .
$$

Here $\Sigma_{2}$ is the string worldsheet, $\gamma_{i j}$ is the induced metric, and we have included a boundary counterterm to regularize the string action.

For the black hole background recall that $B=0$. We then consider a fundamental string $\Sigma_{2} \cong \mathbb{R}^{2}$ wrapping the $\tau$ and $r$ directions, at a point on $\mathbb{H}^{4}$. The powers of the harmonic function $H(r)$ cancel in the integrand, so that

$$
\begin{aligned}
\int_{\Sigma_{2}} X^{-2} \sqrt{\operatorname{det} \gamma} \mathrm{d}^{2} x-\frac{3}{\sqrt{2}} \operatorname{length}\left(\partial \Sigma_{2}\right) & =\lim _{\Lambda \rightarrow \infty} \frac{3}{\sqrt{2}}\left(\int_{r=r_{h}}^{\Lambda} \mathrm{d} r-\sqrt{\frac{9 f(\Lambda)}{2 H(\Lambda)^{3 / 2}}}\right) 2 \pi n \\
& =-\frac{6 \pi n}{\sqrt{2}} r_{h}
\end{aligned}
$$

Identifying $-S_{\text {string }}$ with $\log \langle W\rangle$, we thus find

$$
\log \langle W\rangle_{n}=\frac{1+2 n}{3} \log \langle W\rangle_{n=1} .
$$

Using the identification of the $n$-branched sphere partition function with the squashed sphere result explained in section 1.2, this result agrees with the large $N$ limit of the field theory computation. 


\section{Discussion}

In this paper we have computed the supersymmetric Rényi entropy across an entangling three-sphere for five-dimensional superconformal field theories using localization. In particular we presented a simple argument for why this equals the squashed five-sphere partition function, for appropriate squashing parameters. This argument applies in general dimensions. For a class of $U S p(2 N)$ gauge theories we have constructed the holographic dual 1/2 BPS black hole solution of Euclidean Romans $F(4)$ supergravity. The large $N$ limit of the gauge theory result agrees perfectly with the supergravity computation.

In $[12,13]$ it was conjectured that for any supersymmetric Romans supergravity solution with the topology of $\mathbb{R}^{6}$, with at least $\mathrm{U}(1)^{3}$ isometry, and for which the Killing vector $K$ takes the form $K=b_{1} \partial_{\varphi_{1}}+b_{2} \partial_{\varphi_{2}}+b_{3} \partial_{\varphi_{3}}$, the holographic free energy is

$$
F=\frac{\left(\left|b_{1}\right|+\left|b_{2}\right|+\left|b_{3}\right|\right)^{3}}{27\left|b_{1} b_{2} b_{3}\right|} F_{\mathrm{AdS}_{6}} .
$$

For the explicit 1/2 BPS black hole solution we have found in the present paper, the result (2.37) agrees with this conjecture. Moreover, it was also conjectured that for a BPS Wilson loop wrapping the $\varphi_{i}$ circle, at the origin of the perpendicular $\mathbb{R}^{4}$, one has $\log \langle W\rangle=\frac{\left|b_{1}\right|+\left|b_{2}\right|+\left|b_{3}\right|}{3\left|b_{i}\right|} \log \langle W\rangle_{\mathrm{AdS}_{6}}$. Again, our result (2.40) agrees with this formula.

Notice that the argument in section 1.2 can be applied to any squashed sphere background. In three dimensions, the results of [11] imply that the partition function for an $n$-branched squashed three-sphere, with supersymmetric Killing vector $K=b_{1} \partial_{\varphi_{1}}+b_{2} \partial_{\varphi_{2}}$, is given by the partition function on a different squashed sphere with $\left(b_{1}, b_{2}\right) \rightarrow\left(b_{1}, b_{2} / n\right)$. Here the branch locus is the $S^{1}$ at $\partial_{\varphi_{2}}=0$. We expect a similar result to hold also in five dimensions. It would be interesting to study the implications of this for Rényi entropy computations.

Note added. shortly after submitting this paper to the arXiv we become aware of [6], which has overlap with our work.

\section{Acknowledgments}

The work of L. F. A. and P. R. is supported by ERC STG grant 306260. L. F. A. is a Wolfson Royal Society Research Merit Award holder. J. F. S. is supported by the Royal Society.

Open Access. This article is distributed under the terms of the Creative Commons Attribution License (CC-BY 4.0), which permits any use, distribution and reproduction in any medium, provided the original author(s) and source are credited.

\section{References}

[1] C.G. Callan Jr. and F. Wilczek, On geometric entropy, Phys. Lett. B 333 (1994) 55 [hep-th/9401072] [INSPIRE]. 
[2] P. Calabrese and J.L. Cardy, Entanglement entropy and quantum field theory, J. Stat. Mech. 0406 (2004) P06002 [hep-th/0405152] [INSPIRE].

[3] M. Headrick, Entanglement Renyi entropies in holographic theories, Phys. Rev. D 82 (2010) 126010 [arXiv:1006.0047] [INSPIRE].

[4] H. Casini, M. Huerta and R.C. Myers, Towards a derivation of holographic entanglement entropy, JHEP 05 (2011) 036 [arXiv:1102.0440] [INSPIRE].

[5] T. Nishioka and I. Yaakov, Supersymmetric Rényi Entropy, JHEP 10 (2013) 155 [arXiv: 1306.2958] [INSPIRE].

[6] N. Hama, T. Nishioka and T. Ugajin, Supersymmetric Rényi entropy in five dimensions, JHEP 12 (2014) 048 [arXiv:1410.2206] [INSPIRE].

[7] L.-Y. Hung, R.C. Myers, M. Smolkin and A. Yale, Holographic Calculations of Renyi Entropy, JHEP 12 (2011) 047 [arXiv:1110.1084] [INSPIRE].

[8] J. Kallen, J. Qiu and M. Zabzine, The perturbative partition function of supersymmetric $5 D$ Yang-Mills theory with matter on the five-sphere, JHEP 08 (2012) 157 [arXiv:1206.6008] [INSPIRE].

[9] H.-C. Kim and S. Kim, M5-branes from gauge theories on the 5-sphere, JHEP 05 (2013) 144 [arXiv:1206.6339] [INSPIRE].

[10] N. Hama, K. Hosomichi and S. Lee, SUSY Gauge Theories on Squashed Three-Spheres, JHEP 05 (2011) 014 [arXiv: 1102.4716] [INSPIRE].

[11] L.F. Alday, D. Martelli, P. Richmond and J. Sparks, Localization on Three-Manifolds, JHEP 10 (2013) 095 [arXiv: 1307.6848] [INSPIRE].

[12] L.F. Alday, M. Fluder, P. Richmond and J. Sparks, Gravity Dual of Supersymmetric Gauge Theories on a Squashed Five-Sphere, Phys. Rev. Lett. 113 (2014) 141601 [arXiv:1404.1925] [INSPIRE].

[13] L.F. Alday, M. Fluder, C.M. Gregory, P. Richmond and J. Sparks, Supersymmetric gauge theories on squashed five-spheres and their gravity duals, JHEP 09 (2014) 067 [arXiv: 1405.7194] [INSPIRE].

[14] Y. Imamura, Perturbative partition function for squashed $S^{5}$, arXiv:1210.6308 [INSPIRE].

[15] S. Ferrara, A. Kehagias, H. Partouche and A. Zaffaroni, AdS $S_{6}$ interpretation of $5-D$ superconformal field theories, Phys. Lett. B 431 (1998) 57 [hep-th/9804006] [INSPIRE].

[16] A. Brandhuber and Y. Oz, The D-4 - D-8 brane system and five-dimensional fixed points, Phys. Lett. B 460 (1999) 307 [hep-th/9905148] [INSPIRE].

[17] O. Bergman and D. Rodriguez-Gomez, $5 d$ quivers and their AdS 6 duals, JHEP 07 (2012) 171 [arXiv:1206.3503] [INSPIRE].

[18] D.L. Jafferis and S.S. Pufu, Exact results for five-dimensional superconformal field theories with gravity duals, JHEP 05 (2014) 032 [arXiv:1207.4359] [INSPIRE].

[19] X. Huang, S.-J. Rey and Y. Zhou, Three-dimensional SCFT on conic space as hologram of charged topological black hole, JHEP 03 (2014) 127 [arXiv:1401.5421] [INSPIRE].

[20] T. Nishioka, The Gravity Dual of Supersymmetric Rényi Entropy, JHEP 07 (2014) 061 [arXiv: 1401.6764] [INSPIRE]. 
[21] X. Huang and Y. Zhou, $N=4$ super-Yang-Mills on Conic Space as Hologram of STU Topological Black Hole, arXiv:1408.3393 [INSPIRE].

[22] M. Crossley, E. Dyer and J. Sonner, Super-Rényi entropy \&3 Wilson loops for $\mathcal{N}=4 S Y M$ and their gravity duals, JHEP 12 (2014) 001 [arXiv:1409.0542] [INSPIRE].

[23] L.J. Romans, The F(4) Gauged Supergravity in Six-dimensions, Nucl. Phys. B 269 (1986) 691 [inSPIRE].

[24] M. Cvetič, H. Lü and C.N. Pope, Gauged six-dimensional supergravity from massive type IIA, Phys. Rev. Lett. 83 (1999) 5226 [hep-th/9906221] [INSPIRE].

[25] B. Assel, J. Estes and M. Yamazaki, Wilson Loops in $5 d N=1$ SCFTs and AdS/CFT, Annales Henri Poincaré 15 (2014) 589 [arXiv:1212.1202] [INSPIRE]. 